\title{
MicroRNA-122 Inhibits Lipid Droplet Formation and Hepatic Triglyceride Accumulation via Yin Yang 1
}

\author{
Guo-yi Wua,b Chen Ruic Ji-qiao Chen ${ }^{c}$ Eiketsu Shoc Shan-shan Zhan ${ }^{a, b}$ \\ Xian-wen Yuan ${ }^{a, b} \quad$ Yi-tao Ding ${ }^{a, b}$ \\ aDepartment of Hepatobiliary Surgery, The Affiliated Drum Tower Hospital of Nanjing University \\ Medical School, Nanjing, Jiangsu; ${ }^{b}$ Clinical Medical Center for Digestive Disease of Jiangsu Province, \\ Nanjing, Jiangsu; 'KCI Biotech (Suzhou), Inc., Suzhou, Jiangsu, P.R. China
}

\section{Key Words}

Nonalcoholic Fatty Liver Disease • Fatty Acid/Metabolism • Triglycerides • Cell signaling • Yin Yang 1

\begin{abstract}
Background/Aims: An increase in intracellular lipid droplet formation and hepatic triglyceride (TG) content usually results in nonalcoholic fatty liver disease. However, the mechanisms underlying the regulation of hepatic TG homeostasis remain unclear. Methods: Oil red O staining and TG measurement were performed to determine the lipid content. miRNA expression was evaluated by quantitative PCR. A luciferase assay was performed to validate the regulation of Yin Yang 1 ( $Y Y 1$ ) by microRNA (miR)-122. The effects of miR-122 expression on YY1 and its mechanisms involving the farnesoid $X$ receptor and small heterodimer partner (FXR-SHP) pathway were evaluated by quantitative PCR and Western blot analyses. Results: miR-122 was downregulated in free fatty acid (FFA)-induced steatotic hepatocytes, and streptozotocin and high-fat diet (STZ-HFD) induced nonalcoholic steatohepatitis (NASH) in mice. Transfection of hepatocytes with miR-122 mimics before FFA induction inhibited lipid droplet formation and TG accumulation in vitro. These results were verified by overexpressing miR-122 in the livers of STZ-HFD-induced NASH mice. The 3'-untranslated region (3'UTR) of YY1 mRNA is predicted to contain an evolutionarily conserved miR-122 binding site. In silico searches, a luciferase reporter assay and quantitative PCR analysis confirmed that miR-122 directly bound to the $Y Y 13^{\prime} U T R$ to negatively regulate $Y Y 1$ mRNA in HepG2 and Huh7 cells. The (FXR-SHP) signaling axis, which is downstream of $Y Y 1$, may play a key role in the mechanism of miR-122regulated lipid homeostasis. YY1-FXR-SHP signaling, which is negatively regulated by FFA, was enhanced by miR-122 overexpression. This finding was also confirmed by overexpression of miR-122 in the livers of NASH mice. Conclusions: The present results indicate that miR-122 plays an important role in lipid (particularly TG) accumulation in the liver by reducing YYI mRNA stability to upregulate FXR-SHP signaling.




\section{Introduction}

Nonalcoholic fatty liver disease (NAFLD) is a common liver disease that has become a major public health problem worldwide. NAFLD is characterized by hepatic accumulation of lipids and includes a spectrum of diseases from simple liver steatosis to nonalcoholic steatohepatitis (NASH) with liver dysfunction [1]. NASH may lead to the development of cirrhosis and end-stage liver disease or hepatocellular carcinoma (HCC) [2]. Despite progress in NAFLD research, the pathogenesis of this disease remains unclear and the options for NAFLD diagnosis and treatment are limited.

In mammals, the liver is a metabolic organ that plays an important role in the regulation of TG homeostasis. Excessive TG deposition in hepatocytes derives from an increased delivery of FFA into the liver, leading to the development of NAFLD [3]. In obesity-associated NAFLD, FFA delivery to the liver is increased, especially during the fed state, due to adipose tissue insulin resistance [4]. Accumulated TGs in hepatocytes appear to be relatively inert and associated with benign outcomes; hepatocyte injury is driven by lipotoxicity from FFAs and their derivatives, as well as overloading of mitochondrial capacity [5].

Hepatic lipogenesis is regulated by different pathways, including sterol regulatory element-binding protein 1 (SREBP-1) [6], liver X receptor (LXR) [7], fibroblast growth factor 21 (FGF21) [8], and carbohydrate-responsive element-binding protein (ChREBP) [9]. Previous studies have demonstrated that SREBP-1c is regulated by a nuclear receptor cascade involving the farnesoid X receptor (FXR) and small heterodimer partner (SHP) in obesity and aging $[10,11]$. Notably, a previous study showed that $Y Y 1$ alters lipid metabolic homeostasis via targeting intron 1 of the FXR gene. In addition, FXR is involved in the regulation of insulin resistance and lipid metabolic disorders. FXR inhibits the activation of hepatic stellate cells and inflammatory cell invasion, as well as the regeneration of liver cells to prevent liver fibrosis. Several FXR agonists have been identified and have shown promise in preventing and treating NAFLD, indicating that YY1-FXR may be a therapeutic target in NAFLD.

miRNAs are a class of small non-coding RNAs that regulate gene expression posttranscriptionally by binding to complementary regions in the 3'UTR of target mRNAs, resulting in mRNA degradation or attenuated translation [12]. Several studies have shown that miRNAs play a role in hepatic TG homeostasis. For instance, miR-33 affects TG metabolism by targeting key genes involved in fatty acid oxidation [13]. miR-378 ameliorates hepatic steatosis in obese mice by directly targeting the P110 $\alpha$ subunit, a core component of insulin signaling [14]. miR-122 is one of the most abundant miRNAs in the liver, accounting for approximately $70 \%$ and $52 \%$ of the whole hepatic miRNome in adult mice and humans, respectively $[15,16]$. A recent study showed that miR-122 plays a critical role in the regulation of hepatic TG and cholesterol homeostasis. These data may improve our understanding of the pathogenesis of hepatic metabolic disorders [17, 18]. AMP-activated protein kinase (APK) and circadian metabolic regulators of the peroxisome proliferator-activated receptor family were suggested as putative effectors of miR-122-mediated metabolic control $[15,19]$; however, the molecular mechanisms underlying the regulation of hepatic lipid homeostasis by miR-122 remain unclear.

In this study, we showed that miR-122 was downregulated in steatotic hepatocytes and the livers of STZ-HFD-induced NASH mice. Overexpression of miR-122 in vivo and in vitro inhibited TG accumulation in hepatocytes by destabilizing $Y Y 1$ mRNA to inhibit $Y Y 1$ expression. The FXR-SHP axis was negatively modulated by $Y Y 1$. Our findings reveal a novel post-transcriptional mechanism by which miR-122 regulates $Y Y 1$ expression in the pathogenesis of NAFLD.

\section{Materials and Methods}

\section{Cell culture, transfection, and induction of lipid accumulation in vitro}

Human HCC cell lines HepG2 and Huh7 were purchased from the Cell Bank of the Chinese Academy of Sciences (Shanghai, China). HepG2 and Huh7 cells were grown in DMEM containing 10\% FBS, penicillin (100 


\section{Cellular Physiology Cell Physiol Biochem 2017;44:1651-1664 \begin{tabular}{l|l|l} 
and BOI: 10.1159/000485765 & $\begin{array}{l}\text { C } 2017 \text { The Author(s). Published by S. Karger AG, Basel } \\
\text { www.karger.com/cpb }\end{array}$
\end{tabular}}

Wu et al.: Regulation of Hepatic Triglyceride by MicroRNA-122

$\mathrm{U} / \mathrm{mL})$ and streptomycin $(100 \mathrm{mg} / \mathrm{mL})$ at $37^{\circ} \mathrm{C}$ in a $5 \% \mathrm{CO}_{2}$ humidified incubator. An FFA fat-overloading model was generated by mixing oleic acid and palmitate acid obtained from Sigma-Aldrich (St. Louis, MO, USA) in a 2:1 ratio to establish a lipid accumulation model to induce hepatocytes as reported previously [20]. This model was shown to mimic NAFLD characteristics, with low levels of cellular toxicity [21]. Control groups in all cases were treated with 1\% fatty acid-free BSA without palmitate acid and oleic acid. Three hours prior to FFA treatment, the oligonucleotides were transiently transfected into hepatocytes. The miR122 mimic (miR-122) and the corresponding negative control (miR-N.C.) were purchased from GenePharma (Shanghai, China). Transfection was performed using Lipofectamine ${ }^{\mathrm{TM}} 2000$ Transfection Reagent from Life Technologies Corporation (Carlsbad, CA, USA) according to the manufacturer's instructions.

In the miR-122 mimic group, synthetic pre-miR-122 (miRIDIAN mimic, has-miR-122, UGGAGUGUGACAAUGGUGUUUG) was transfected into hepatocytes; in the miR-N.C. group, both cell lines were transfected with pre-miR-122 negative control (miRIDIAN miR mimic transfection negative control, N.C.).

\section{Mouse NASH model and miR-122 over-expression vector treatment}

The DNA fragment for mmu-miR-122 was amplified from genomic DNA and inserted into the AgeI/ EcoRI site of the lentiviral expression vector pGCSIL-GFP from GeneChem (Shanghai, China) and verified by sequencing. All animal procedures were performed according to the "Guide for the Care and Use of Laboratory Animals" prepared by the National Academy of Sciences and published by the National Institutes of Health (NIH publication 86-23, revised 1985). The experimental protocol was approved by the Animal Care Ethics Committee of Nanjing Drum Tower Hospital. Mice were randomly divided into four groups (eight mice/group): a normal control group, a NASH model group, a miR-122 over-expression group, and an empty vector treatment group. Normal control mice were housed without any treatment and fed a normal diet (composed of $12 \mathrm{kcal} \%$ fat). NASH model mice were generated as previously described [22]. Briefly, pregnant C57BL/6J mice were purchased from the Laboratory Animal Center of Shanghai, Academy of Science. The new mice were subjected to a single subcutaneous injection of $200 \mu \mathrm{g}$ STZ from Sigma-Aldrich on the second day after birth. Four weeks after STZ injection, male mice were fed a HFD (composed of 60 $\mathrm{kcal} \%$ fat) ad libitum until sacrifice. One week after beginning the HFD, normal control and NASH model mice were injected with $0.2 \mathrm{~mL}$ physiological saline via the tail vein every 3 days for a total of five times. miR-122 overexpression mice received $0.2 \mathrm{~mL}$ lentiviral miR-122 over-expression vector $\left(1 \times 10^{7} \mathrm{IU} / \mathrm{mL}\right)$ and empty vector treatment mice received $0.2 \mathrm{~mL}$ empty lentiviral vector $\left(1 \times 10^{7} \mathrm{IU} / \mathrm{mL}\right)$ at the same time points as the normal control mice receiving saline injection via the tail vein. At the end of week 8 , the mice in each group were euthanized, the livers were removed and a portion was immediately frozen and stored at $-80^{\circ} \mathrm{C}$ until subsequent analyses, including TG content analysis and quantitative PCR. The remaining portion of the liver was fixed with $10 \%$ formalin for subsequent liver histology evaluation.

\section{Analysis of lipid content and cellular TG measurement}

Cellular lipid droplets were visualized by Oil Red 0 staining as described previously [23]. Briefly, cells on slides were fixed in 10\% formalin from WAKO Pure Chemical Industries (Osaka, Japan) at $4^{\circ} \mathrm{C}$ for $10 \mathrm{~min}$ and the slides were rinsed with PBS (pH 7.4). After air drying, the slides were placed in 100\% propylene glycol for $2 \mathrm{~min}$ and stained with a $0.5 \%$ Oil Red 0 solution in propylene glycol for $30 \mathrm{~min}$. The slides were transferred to an $85 \%$ propylene glycol solution for $1 \mathrm{~min}$, rinsed in distilled water twice, and processed for hematoxylin counterstaining.

Tissue and cellular TG were extracted and quantified with a TG determination kit. In brief, TG content was measured using the Triglyceride Assay Kit from NanJing JianCheng Bioengineering Institute (Nanjing, Jiangsu, China) according to the manufacturer's instructions and normalized to total protein concentration. Intracellular TG content is expressed in $\mu \mathrm{g} / \mu \mathrm{L}$ protein and $\mathrm{TG}$ content in mice liver tissue is expressed in $\mathrm{mg} / \mathrm{g}$.

\section{RNA extraction and quantitative reverse transcription PCR ( $q R T-P C R$ )}

Total RNA including miRNA was isolated from cultured cells and tissues using TRIzol Reagent from Life Technologies Corporation (Carlsbad, CA, USA) following the manufacturer's instructions. Total RNA was quantified using a spectrophotometer from BioTek (Shoreline, WA, USA) at $260 \mathrm{~nm}$. Total RNA (2 $\mu \mathrm{g})$ was reverse-transcribed into cDNA using SuperScript III from Life Technologies Corporation (Carlsbad, CA, USA) 
according to the manufacturer's instructions. For the detection of miR-122 levels, qRT-PCR was performed using an ABI ViiA ${ }^{\mathrm{TM}} 7$ System (Applied Biosystems, Foster City, CA, USA) and TaqMan microRNA assay kits with specific primers for miR-122 from Applied Biosystems (Foster City, CA, USA) and endogenous U6 snRNA as the control. For the detection of FXR and SHP expression, qRT-PCR was performed using Fast SYBR Green Master Mix on an ABI ViiA 7 System. The primers for YY1, FXR, SHP, and GAPDH (control) used in this study are available upon request.

\section{Cell viability MTT assay}

Cell viability was determined by the MTT assay using tetrazolium 3-(4, 5) -dimethylthiahiazo(-z-y1)-3, 5 -di-phenytetrazoliumromide dye. Briefly, approximately $5 \times 10^{3}$ cells per well were seeded into a 96-well plate. At the indicated times, $20 \mathrm{~mL}$ MTT solution were added to each well and the plate was subsequently incubated at $37^{\circ} \mathrm{C}$ for $4 \mathrm{~h}$ in the incubator. The liquid was then removed from the plate and $150 \mathrm{~mL}$ DMSO were added to each well. All plates were read at $490 \mathrm{~nm}$.

\section{Bioinformatics and luciferase reporter assay}

An in silico search for possible miRNA-binding sites in the 3' untranslated region (UTR) of the YY1 gene was performed using TargetScan and miRANDA [24] from the Memorial Sloan-Kettering Cancer Center (New York, NY, USA).

To examine the YY1 3'UTR as a target of miR-122 in vitro, luciferase assays were performed with a pmirGLO Dual-Luciferase miRNA Target Expression Vector from Promega (Madison, WI, USA) containing both the coding sequences of firefly luciferase and Renilla luciferase (internal control). The YY1 3'UTR (accession number NG_046908.1) was cloned into the pmiRGLO vector system using specific primers (Fig. 4A). Plasmids were verified by Sanger sequencing. $293 \mathrm{~T}$ cells were seeded into 12 -well plates $\left(5 \times 10^{5}\right.$ cells/well). After $24 \mathrm{~h}$, the cells were transfected with 200 nM hsa-miR-122 miRNA mimics or a negative control (hsa-miR-122, Negative Control) and $1.6 \mu \mathrm{g} /$ well plasmid DNA with the use of Lipofectamine 2000 (Invitrogen). The activities of firefly and Renilla luciferase were measured $24 \mathrm{~h}$ after cotransfection using the DualLuciferase Reporter 1000 Assay System from Promega (Madison, WI, USA) according to the manufacturer's protocol. HsamiR-122 was used as a positive control. Analysis was performed using the GloMax Multi Detection System from Promega (Madison, WI, USA). YY1-MUT was used to introduce mutations into the miR-122-binding sites using specific primers (Table 1). Three independent luciferase reporter assays were performed including wild-type and mutated target sequences.

Table 1. Primer sequences used for subcloning and mutagenesis

\begin{tabular}{|c|c|c|}
\hline $\begin{array}{l}\text { Oligonucle } \\
\text { otide }\end{array}$ & Sequence $\left(5^{\prime}-3^{\prime}\right)$ & Purpose \\
\hline YY1-WT-F & $\begin{array}{l}\text { CTCATCTCTTCTGTCCTTTCCTGTCTCTGAAATAGTCATCACTCCCCTTGACTCTCTCTG } \\
\text { TTCACGTCTC }\end{array}$ & $\begin{array}{c}\text { Subcloni } \\
\text { ng of the } \\
\text { YY1 } 3^{\prime} \\
\text { end into } \\
\text { pmirGlo }\end{array}$ \\
\hline YY1-WT-R & $\begin{array}{l}\text { TCGAGAGACGTGAACAGAGAGAGTCAAGGGGAGTGATGACTATTTCAGAGACAGGAAA } \\
\text { GGACAGAAGAGATGAGAGCT }\end{array}$ & $\begin{array}{c}\text { Subcloni } \\
\text { ng of } \\
\text { the YY1 } 3 \\
\text { 'end into } \\
\text { pmirGlo }\end{array}$ \\
\hline YY1-MUT-F & $\begin{array}{l}\text { CTCATCTCTTCTGTCCTTTCCTGTCTCTGAATATGTGTAGTGAGGCCTTGACTCTCTCT } \\
\text { GTTCACGTCTC }\end{array}$ & $\begin{array}{c}\text { Subcloni } \\
\text { ng of the } \\
\text { YY1 } 3^{\prime} \mathrm{M} \\
\text { UT end } \\
\text { into } \\
\text { pmirGlo }\end{array}$ \\
\hline YY1-MUT-R & $\begin{array}{l}\text { TCGAGAGACGTGAACAGAGAGAGTCAAGGCCTCACTACACATATTCAGAGACAGGAAA } \\
\text { GGACAGAAGAGATGAGAGCT }\end{array}$ & $\begin{array}{c}\text { Subcloni } \\
\text { ng of the } \\
\text { YY1 } 3^{\prime} \mathrm{M} \\
\text { UT end } \\
\text { into } \\
\text { pmirGlo }\end{array}$ \\
\hline YY1-PC-F & CCAAACACCATTGTCACACTCCAACCGGTCAAACACCATTGTCACACTCCAC & $\begin{array}{l}\text { Subcloni } \\
\text { ng of the } \\
\text { YY1 } 3^{\prime} P C \\
\text { end into } \\
\text { pmirGlo }\end{array}$ \\
\hline YY1-PC-R & $\begin{array}{l}\text { TCGAGTGGAGTGTGACAATGGTGTTTGACCGGTTGGAGTGTGACAATGGTGTTTGGAG } \\
\text { CT }\end{array}$ & $\begin{array}{c}\text { Subcloni } \\
\text { ng of } \\
\text { the YY1 } 3 \\
\text { 'PC end } \\
\text { into } \\
\text { pmirGlo }\end{array}$ \\
\hline
\end{tabular}


Western blotting

Extracted protein from cells was separated by 10\% SDS-PAGE and transferred to nitrocellulose membranes at $100 \mathrm{~mA}$ for $2 \mathrm{~h}$. The membranes were blocked in skimmed milk for $1 \mathrm{~h}$ at room temperature and overnight at $4^{\circ} \mathrm{C}$ in anti-FXR and anti-SHP $(1: 1,000)$ antibodies from Abcam (Cambridge, UK), followed by incubation with secondary antibodies at room temperature for $2 \mathrm{~h}$. The immunoreactive bands were visualized using an ECL-PLUS Kit from Thermo Scientific (Piscataway, NJ, USA). The relative protein expression levels were normalized to that of GAPDH.

\section{Histological examination}

Liver sections were prepared as described previously [25], stained with H\&E and observed for hepatic steatosis on an Olympus microscope. The steatosis score was calculated to semi-quantitatively evaluate the severity of hepatic steatosis as previously reported [26]. Briefly, the steatosis grade was scored according to the degree of parenchymal involvement as follows: $0,<5 \% ; 1,5 \%$ to $33 \% ; 2,33 \%$ to $66 \%$; and $3,>66 \%$.

\section{Statistical analysis}

Values are expressed as the mean \pm standard error of the mean (SEM) or mean \pm standard deviation (SD). Significant differences between two groups were determined by Student's $t$ test. Statistical significance is displayed as $* \mathrm{P}<0.05$, ${ }^{* *} \mathrm{P}<0.01$ or $* * * \mathrm{P}<0.001$.

\section{Results}

Effect of FFA on lipid accumulation in HepG2 and Huh7 cells

Lipid accumulation and increased numbers of lipid droplets are the hallmarks of NAFLD. The overwhelming influx of FFA may cause hepatotoxicity and stimulate progression from simple steatosis to NASH via several mechanisms beyond direct cytotoxicity. To gain insight into the underlying mechanism, HepG2 and Huh7 cells were exposed to FFA. HepG2 and Huh7 cells were cultured and induced with $0.5 \mathrm{mM}$ FFA for $24 \mathrm{~h}$ before measuring total lipids using Oil Red 0 dye or TG content using a TG testing kit. As shown in Fig. 1A, lipid droplet accumulation in FFA-treated cells indicated lipid accumulation in both HepG2 and Huh 7 cells as a model of NAFLD. The basal TG accumulation level was low in both HepG2 and Huh7 cells; however, cells exposed to FFA showed a high accumulation of TG, suggesting that a successful in vitro NAFLD model was generated using 0.5 mM FFA (Fig. 1B).

\section{Differential expression of miR-122 and FXR in steatotic hepatocytes}

To investigate any potential correlation between miR-122 expression and lipid metabolic homeostasis in the fatty liver, miR-122 expression was measured in different cells following FFA treatment by quantitative real-time PCR. The mature miR-122 levels in HepG2 and Huh7 steatotic hepatocytes were significantly decreased by $21 \%$ and $26 \%$ (Fig. 2A), respectively, compared with those in untreated cells. Since $Y Y 1$ is a regulator of FXR, the YY1 mRNA level was analyzed by quantitative-PCR. Cells exposed to FFA showed a significant upregulation of the YY1 mRNA level in both cell lines (Fig. 2B). To investigate the regulation of FXR by FFA, FXR and its target gene, SHP, were examined by Western blotting. As shown in Fig. 2C, the expression of FXR and SHP was significantly downregulated in both hepatic steatotic cell lines compared to non-steatotic cells.

\section{miR-122 regulates lipid droplet formation and TG content in HepG2 and Huh7 cells}

To examine the regulation of lipid deposition by miR-122, Oil red 0 staining and TG content analysis were performed in steatotic HepG2 and Huh7 cells treated with or without miR-122 mimics. The MTT assay showed no statistically significant differences in OD values at $24 \mathrm{~h}$ between steatotic cells treated with miR-122 mimics at concentrations below $100 \mathrm{pM}$ and the N.C. and control groups (all P > 0.05) (Fig. 3A). These results indicate that treatment with 100 pM miR-122 mimics has no effect on the viability of steatotic HepG2 and Huh7 cells. Basal lipid droplet and TG accumulation levels were high in steatotic HepG2 and Huh7 


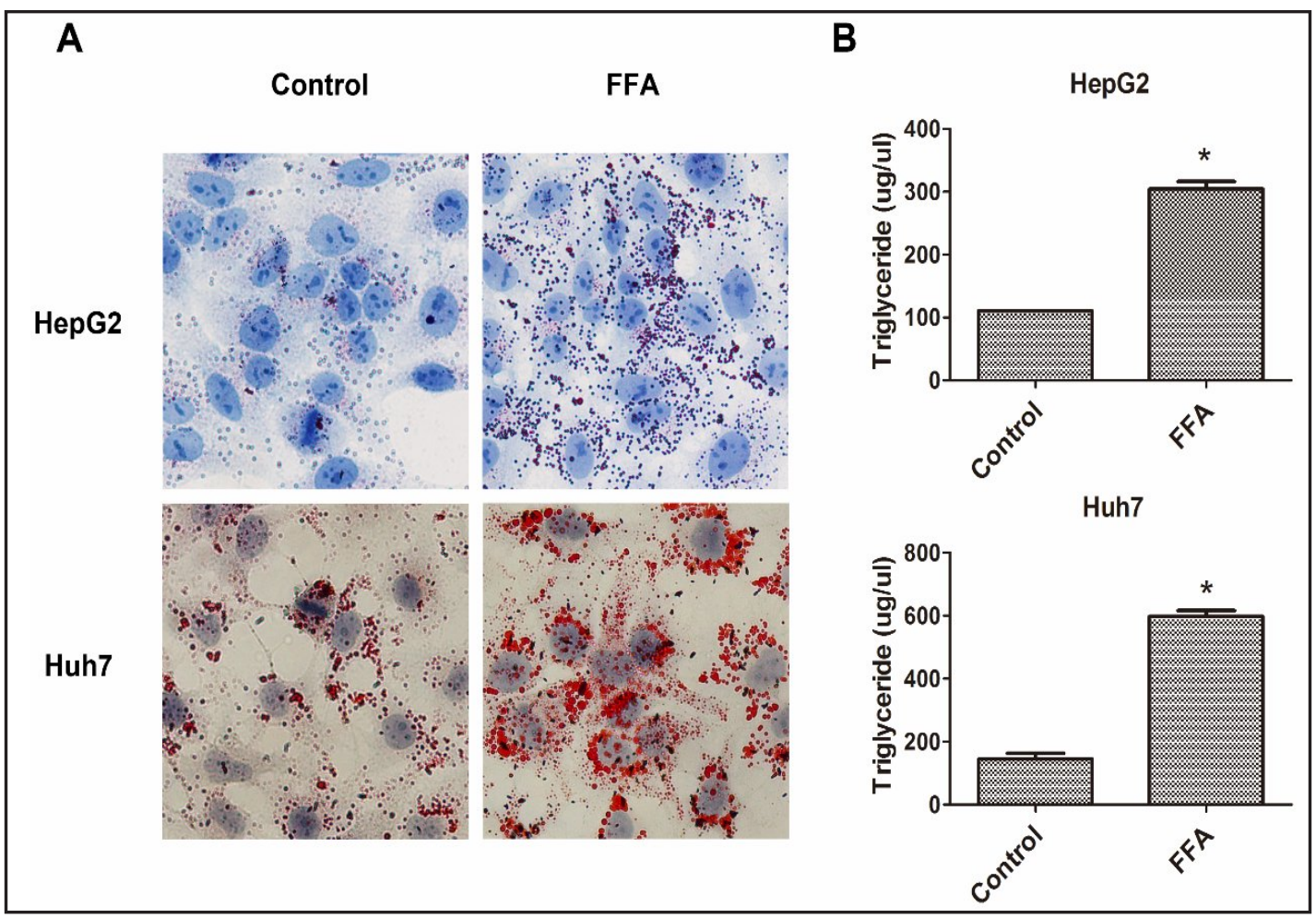

Fig. 1. Lipid accumulation in HepG2 and Huh7 cells treated with $0.5 \mathrm{mM}$ FFA for $24 \mathrm{~h}$. (A) Representative photomicrographs of HepG2 and Huh7 cells $(\times 40)$ stained with Oil Red O. (B) TG content in HepG2 and Huh7 cells. The results are expressed as the mean \pm SEM of three independent experiments. *Significant effect of FFA-treated vs. untreated HepG2 or Huh7 cells.

cells, which were used as the model control (Fig. 3B and C). As shown in Fig. 3B and C, lipid droplet formation and TG levels were significantly lower in the miR-122 treatment group than in the model control and N.C. groups $(\mathrm{P}<0.05)$. Treatment with miR-122 mimics therefore suppressed lipid droplet formation and reduced TG content in both HepG2 and Huh7 cells in the presence of FFA, indicating that miR-122 mimics negatively regulate lipid droplet formation in hepatocytes when the level of lipid accumulation is high.

Confirmation of the YY1 3'UTR as a target of miR-122 by luciferase reporter assays

The software tools miRANDA and TargetScan predicted the binding of miR-122 to YY1 3'UTR transcript positions 615-616, 618-621 and 623-628 (position relative to the translational stop codon, NG_046908.1, Fig. 4A), with mirSVR and PhastCons scores of -0.1655 and 0.5237 , respectively. Co-transfection of miR-122 mimics with the YY1 3'UTR target sequence into $293 \mathrm{~T}$ cells resulted in a $63 \%$ decrease in luciferase activity compared with the negative control $(\mathrm{P}<0.01)$. In contrast, luciferase activity remained unaffected by co-transfection of the YY1 3'UTR target sequence carrying the miR-122 binding sites in the mutated form compared with the wild-type construct (Fig. 4B), indicating a negative interaction between miR-122 and the predicted binding sites in the YY1 3'UTR.

miR-122 mimics regulate YY1 and FXR levels in HepG2 and Huh7 cells

Our data and those of recent reports $[15,17]$ suggest that miR-122 regulates lipid accumulation and TG homeostasis. It was reported that $Y Y 1$ suppresses FXR transcription by binding to the $Y Y 1$ responsive element in intron 1 of the FXR gene. The downregulation of FXR and miR-122 expression in FFA-induced HepG2 cells led us to speculate that miR-122 may regulate FXR signaling by suppressing $Y Y 1$ mRNA. To test this hypothesis, the relative abundance of $Y Y 1$, FXR, and SHP was examined in HepG2 and Huh7 cell lines by quantitative 
Fig. 2. FFA downregulates miR-122 expression and suppresses the expression of FXR and SHP. (A) HepG2 and Huh7 cells were treated with $0.5 \mathrm{mM}$ FFA or vehicle DMSO for $24 \mathrm{~h}$ and the expression of mature miR-122 was measured by quantitativePCR. (B) The YY1 mRNA level in HepG2 and Huh7 cells was analyzed by quantitative-PCR. (C) The expression of FXR and SHP in HepG2 and Huh7 cells was analyzed by Western blotting after $0.5 \mathrm{mM}$ FFA treatment for $24 \mathrm{~h}$.
A

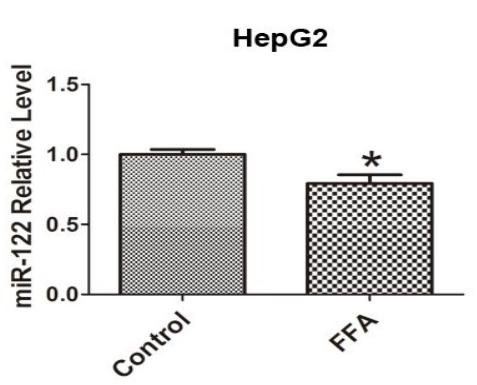

B

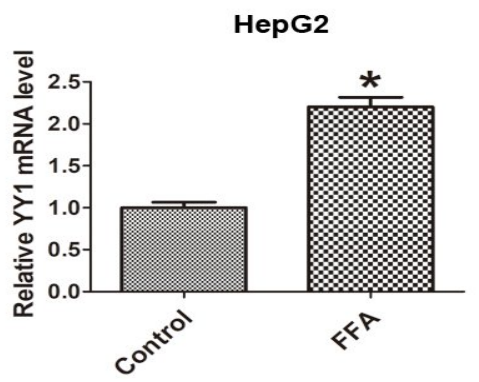

C

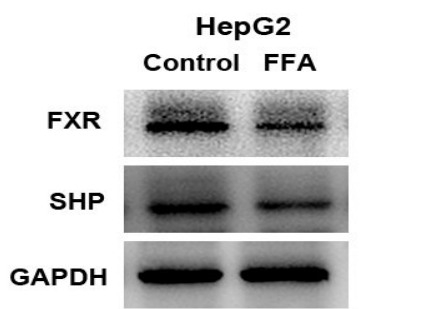

Huh7

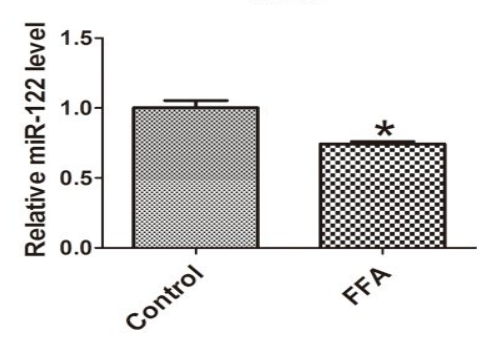

Huh7
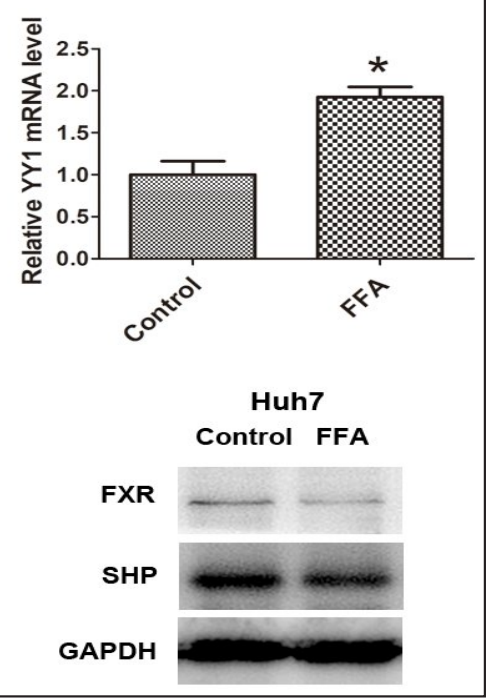

real-time PCR and Western blotting, respectively (Fig. 5). Quantitative real-time PCR was performed to confirm the effect of miR-122 mimics on $Y Y 1$ in both steatotic hepatic cell lines. Transfection of 100 pM miR-122 mimics significantly decreased the abundance of YY1 mRNA in steatotic HepG2 and Huh7 cells. Transfection of miR-122 mimics into steatotic hepatocytes upregulated FXR and SHP protein expression in both steatotic cell lines. The negative control had no effect on mature miR-122 levels or FXR protein expression.

miR-122 inhibits hepatic TG accumulation and improves the histology of NASH mice

To further evaluate the effect of miR-122 on hepatic steatosis in vivo, STZ-HFD-induced $\mathrm{NASH}$ mice were generated to induce insulin resistance and liver steatosis as observed in previous reports [22]. We adopted the 'lentiviral tail vein injection' method to deliver premmu-miR-122 into mouse hepatocytes. C57BL/6 mice were treated with $200 \mu \mathrm{L}$ saline, miR$122\left(1 \times 10^{7} \mathrm{TU} / \mathrm{mL}\right.$ of the lentiviral miR-122 vector), or the empty lentiviral vector as a N.C. for a total of five times. A NASH mouse model developed after 4 weeks of HFD feeding. The observed alteration in hepatic steatosis was confirmed by H\&E staining and TG content was analyzed as described previously. Examination of H\&E-stained liver sections revealed fatty degeneration, inflammatory cell infiltration, and hepatocellular ballooning predominantly around the central veins in mice liver tissue from the vehicle group. The steatosis score was significantly higher in STZ-HFD mice than in the normal control group (Fig. 6A). The steatosis score was significantly lower in the lentiviral miR-122 injection group compared with the STZ-HFD mice group or the empty lentivirus injection group. The steatosis scores in all groups are shown in Table 2. The overexpression of miR-122 resulted in a decrease in TG content (Fig. 6B). Empty lentiviral vector-injected mice showed no significant difference in the histology or TG content in the livers of NASH mice. 
Fig. 3. miR-122 mimics suppress lipid accumulation in FFA-treated HepG2 and Huh7 cells. (A) The effect of miR-122 on FFA-treated HepG2 and Huh7 cell viability was evaluated using the MTT assay. FFA (0.5 mM)-treated HepG2 and Huh7 cells were transfected with different concentrations of miR-122 mimics and a scramble control. The rate of cell inhibition was measured $24 \mathrm{~h}$ post-transfection using the MTT assay. Data are presented as the mean \pm SEM $(\mathrm{n}=$ 3). (B, C) Two hours after transfection with 100 pM miR-122 mimic or N.C., HepG2 or Huh7 cells were treated with FFA $(0.5 \mathrm{mM})$ for 24 h. Lipid synthesis was examined by Oil Red 0 staining (40x). The inhibitory effect of miR-122 on lipid accumulation in both cell lines is shown. TG content was analyzed in HepG2 and Huh7 cells. The results are expressed as the mean \pm SEM of three independent experiments. *Significant effect of miR-122-treated vs. untreated HepG2 or Huh7 cells.
B
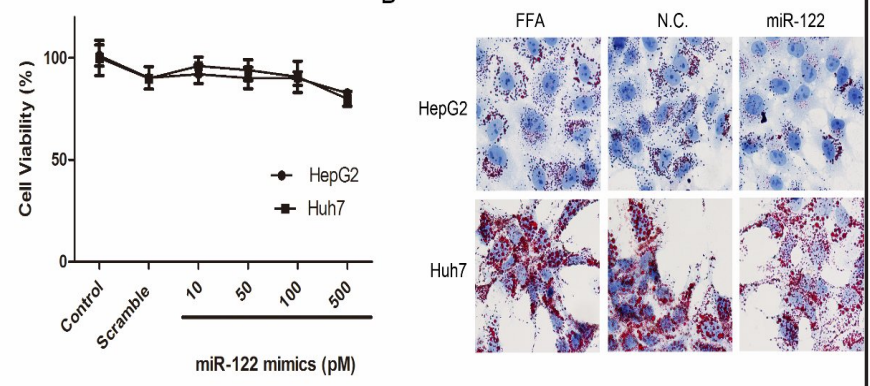

C
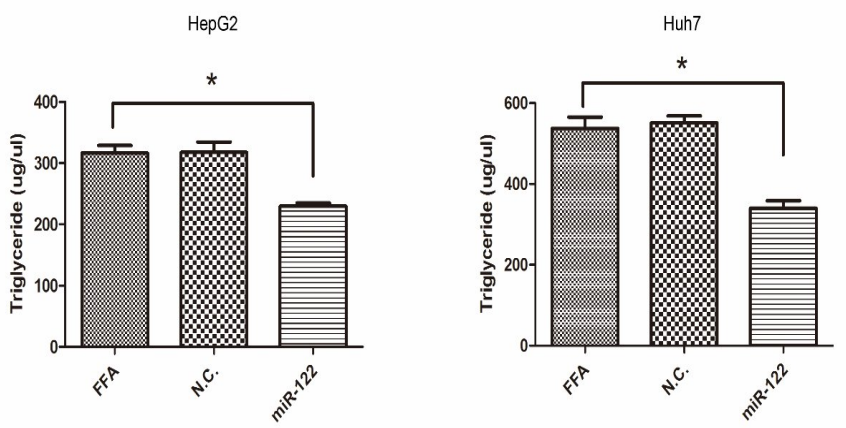

Fig. 4. The YY1 3'UTR is a direct target of miR-122. (A) YY1-luciferase reporter plasmid construction. The seed sequence of miR-122 targeting YY1 is indicted in gray. Mutant sequence of the reporter plasmid is indicted. (B) $293 \mathrm{~T}$ cells were transfected with a firefly luciferase transcript containing either the wild-type or mutant form of the YY1 3'UTR and transfected with either the normal control (N.C.) or miR-122 mimics and assessed for luciferase activity $48 \mathrm{~h}$ post-transfection.

Overexpression of miR122 regulates $Y Y 1, F X R$ and SHP levels in NASH mice

Quantitative real-time PCR assays were performed to analyze the mRNA levels of mature miR-122, $Y Y 1$, $F X R$, and SHP in liver tissues.

As shown in Fig. 7, miR-122 expression levels were reduced in NASH mice. Mature miR-
A
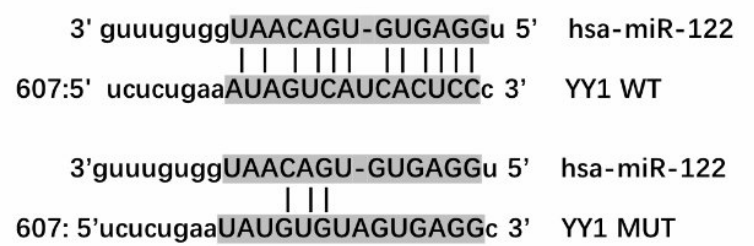

B

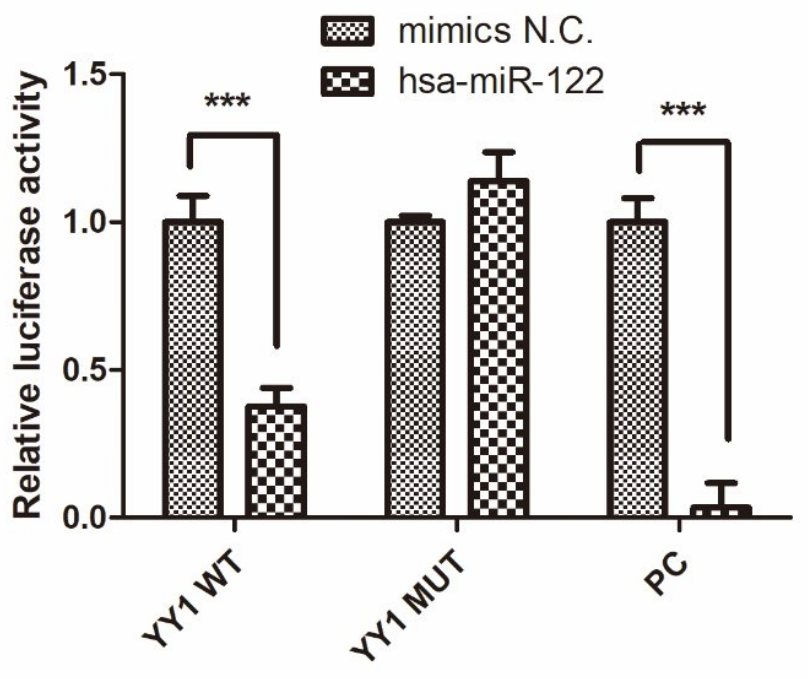


Fig. 5. miR-122 mimics decrease YY1 mRNA levels in the plasma and upregulate FXR-SHP. HepG2 or Huh7 cells were transfected with $100 \mathrm{pM}$ miR-122 mimics or N.C. for $2 \mathrm{~h}$ and then treated with FFA $(0.5 \mathrm{mM})$ for 24 h. (A) YY1 mRNA levels were determined using quantitative realtime PCR. Significant differences between the FFA model and miR-122 mimics-transfected cells are shown. $\mathrm{P}<0.001$. (B) FXR and SHP protein levels after treatment with miR-122 mimics or FFA were analyzed by Western blotting.

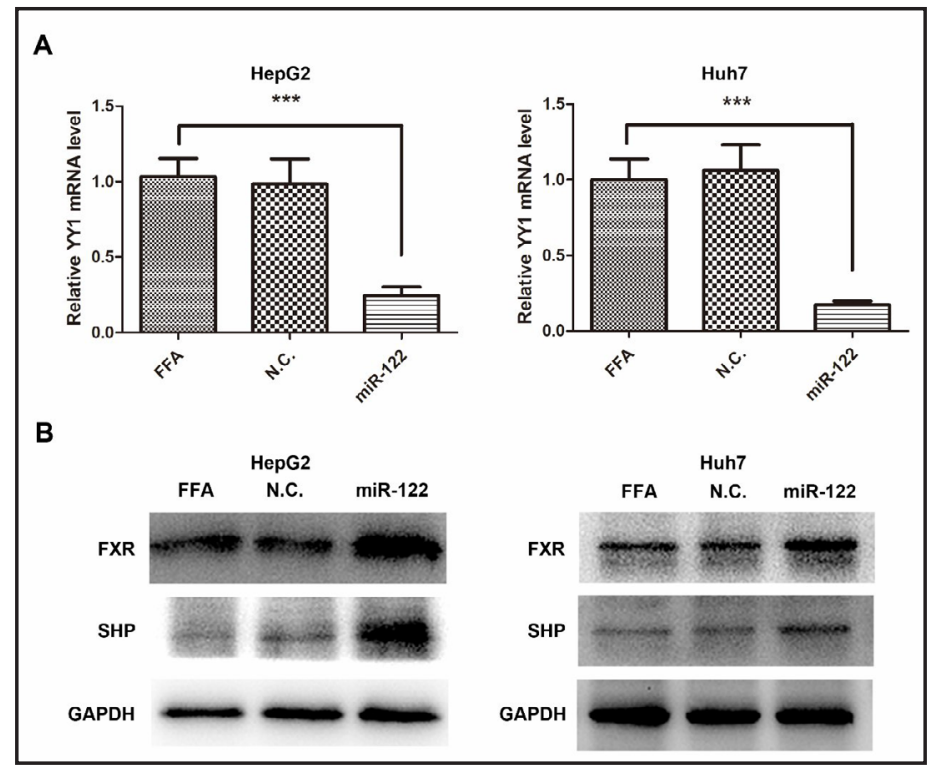

Fig. 6. Lipid deposition and triglyceride content in liver tissues after 8 weeks. (A) H\&E staining of hepatocytes $(\times 40)$ showing hepatocytes with enlarged fat vacuoles. a. Mice fed a normal diet were used as a control. b. Mice fed a HFD after STZ injection showed enlarged fat vacuoles in almost all hepatocytes. The vacuoles pushed the nucleus to the periphery of the cell, presenting the characteristic signet ring appearance, which indicates the successful development

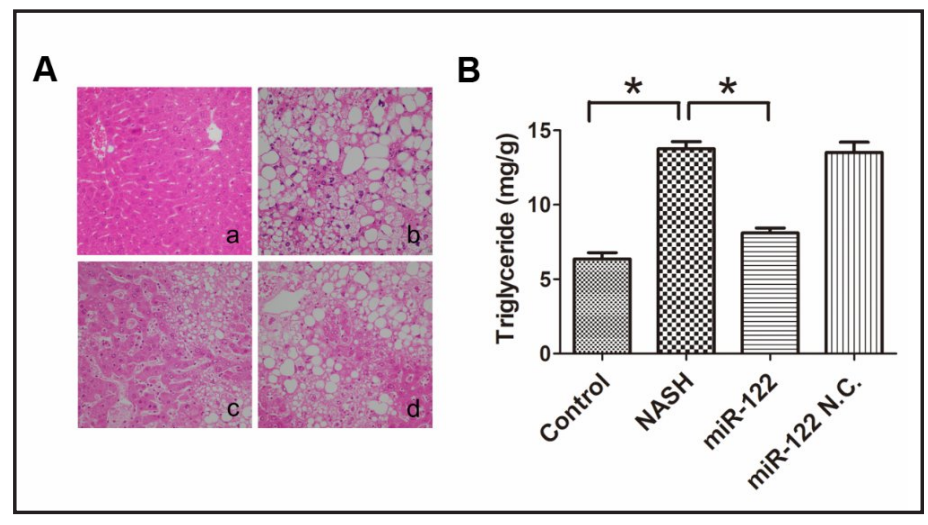
of the NASH mouse model. c. Histogram of the livers of NASH mice with miR-122 overexpression showing the smaller size of fat vacuoles. d. Histogram of the livers of NASH mice treated with the empty lentiviral vector showing that the size of fat vacuoles did not change significantly compared with the NASH model group. (B) Hepatic TG content of mice treated with the miR-122 overexpression vector, the empty lentiviral vector (miR-122 normal control), saline only (NASH model), or normal diet mice (control; $\mathrm{n}=8$ ). Data represent the mean \pm SEM $*$ Significant differences between the control and NASH groups, as well as between the NASH and miR-122 groups are shown. $\mathrm{P}<0.05$.

122 levels were elevated in the livers of model mice infected with the lenti-miR-122 virus, whereas empty lentivirus injection had no effect on mature miR-122 levels. To assess the effect of miR-122 overexpression on $Y Y 1$ and its downstream FXR and SHP mRNA levels in vivo, $Y Y 1, F X R$ and SHP mRNA levels were examined by quantitative real-time PCR. Consistent with the in vitro results, $Y Y 1$ mRNA levels were upregulated, whereas FXR and SHP mRNA levels were downregulated in NASH mouse livers
Table 2. NAFLD steatosis scores. Data are shown as the mean \pm SD

\begin{tabular}{lc}
\hline & Steatosis score \\
\hline Control & 0 \\
NASH & $2.75 \pm 0.46$ \\
miR-122 & $1.88 \pm 0.83$ \\
miR-122 N.C. & $2.50 \pm 0.53$ \\
\hline
\end{tabular}


A

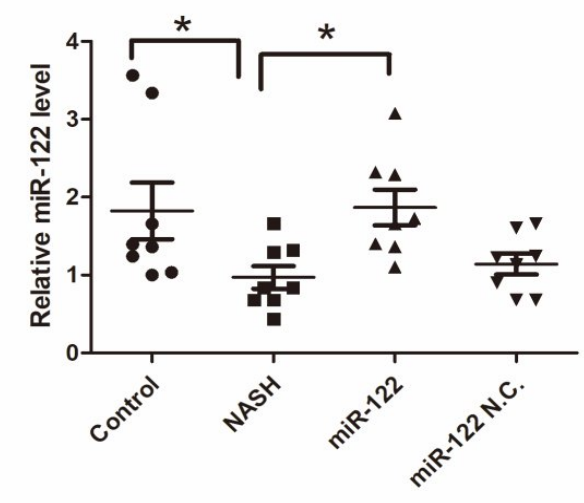

C

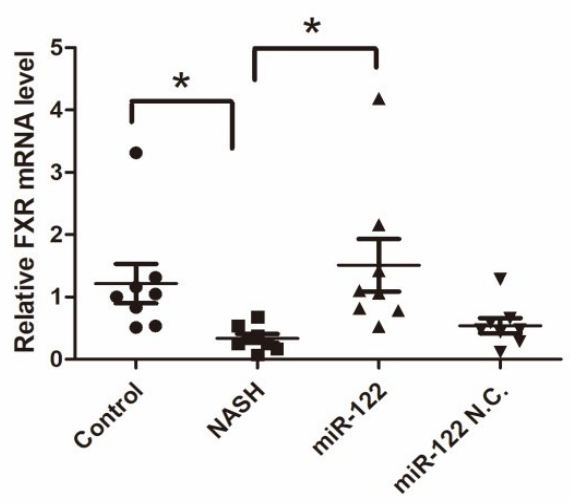

B

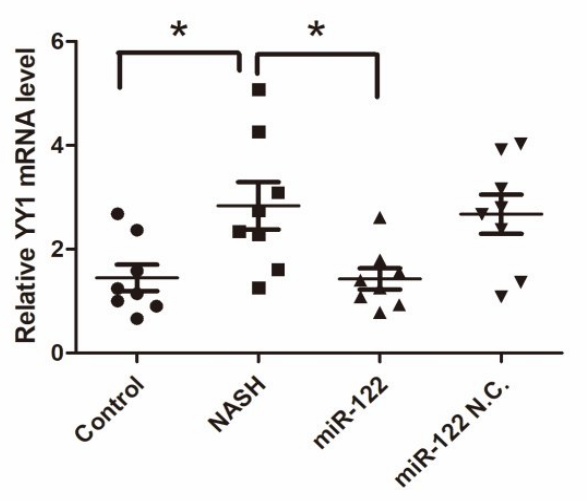

D

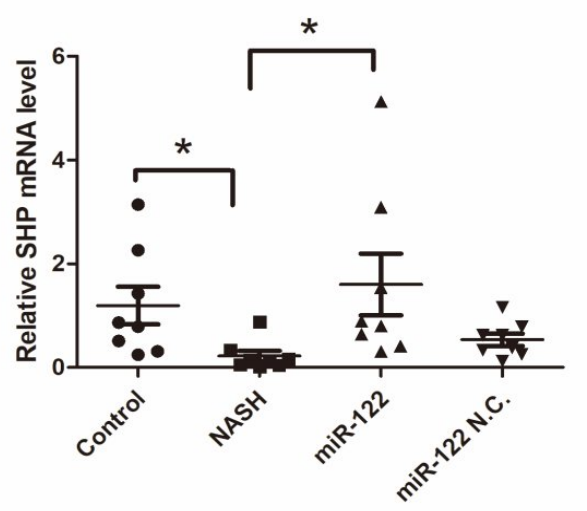

Fig. 7. Relative gene expression in mouse liver tissues after lentiviral miR-122 injection. (A) Mature miR122 was upregulated after lentiviral miR-122 injection. miR-122 regulated the expression of YY1 (B), FXR $(C)$, and SHP (D) mRNAs in mouse liver tissues $(n=8)$. Data are expressed as the mean \pm SEM * Significant differences between the control and NASH groups, and between the NASH and miR-122 groups are shown. $\mathrm{P}<0.05$.

compared with those in wild-type healthy mice. In agreement with the in vitro results, $Y Y 1$ mRNA levels were downregulated, whereas FXR and SHP mRNA levels were significantly upregulated in lenti-miR-122-injected mice. No changes were observed in the empty lentivirus-injected group. These results further suggest that miR-122 is involved in the regulation of the YY1-FXR-SHP signaling pathway in vivo.

\section{Discussion}

NAFLD is a common chronic liver disease worldwide and a major public health concern in modern society [27]. However, liver steatosis is a complex process modulated by many factors and much of its pathogenesis remains undiscovered. Several studies have shown that miR-122 plays a role in NAFLD $[15,17]$. In this study, we elucidated a novel mechanism underlying the effect of miR-122 and identified the YY1-FXR-SHP regulatory axis as a potential target of miR-122 with a protective effect against hepatic steatosis.

Studies have shown that miRNAs play a critical role in the suppression or stimulation of hepatic lipogenesis. Furthermore, miR-122 is the most abundant miRNA in the liver [28]. miR-122 plays a central role in liver development, differentiation, homeostasis, and other functions. miR-122 expression is driven by liver-enriched transcription factors (LETFs), including hepatocyte nuclear factor (HNF) 6 and 4a [29-31]. miR-122 plays a crucial role in the regulation of cholesterol and fatty acid metabolism in the adult liver. In vivo antisense 
studies coupled with microarray analysis have been instrumental to uncover the role of miR122 in lipid metabolism. Indeed, antisense-mediated inhibition of hepatic miR-122 markedly lowered plasma cholesterol levels in both mice and non-human primates [15, 16]. Despite reports in which miR-122 was downregulated in a steatotic hepatocyte model and miR122 mimics reduced hepatocyte steatosis [18], the mechanism by which miR-122 regulates hepatic lipogenesis remains unknown. Many studies have attempted to identify downstream targets of miR-122 and found that miR-122 induced changes in lipogenic gene expression, including fatty acid synthesis, TG synthesis, fatty acid oxidation and transport. One of the studies demonstrated that miR-122 inhibition in a diet-induced mouse model of obesity resulted in decreased mRNA expression levels of acetyl-CoA carboxylase 2, FASN, stearoyl CoA desaturase-1, and HMG CoA reductase [15]. FXR agonists were reported to upregulate miR-122, suppressing the growth of hepatocellular carcinoma cells [32]. Therefore, we hypothesized there may be a close relationship between miR-122 and the FXR signaling axis.

FXR, or NR1H4, is a member of the nuclear receptor superfamily. The importance of FXR in TG metabolism was confirmed in FXR-deficient mice, which exhibited marked hepatosteatosis and hypertriglyceridemia [33]. The TG-lowering effects of endogenous and synthetic FXR agonists were evaluated in rodent models [10]. The synthetic FXR agonist GW4064 was able to prevent liver steatosis in obese mice, such as the ob/ob and db/db models [34]. Based on this finding, obeticholic acid (OCA) was proposed for the treatment of NASH as a synthetic FXR agonist [35]. A phase 2 trial showed that administration of OCA at $25 \mathrm{mg}$ or $50 \mathrm{mg}$ daily for 6 weeks reduced markers of liver inflammation and fibrosis and increased insulin sensitivity. However, lipid abnormalities were associated with the use of OCA for NASH treatment, giving rise to safety concerns [36]. Therefore, the identification of miR-122 as an FXR regulator in the modulation of lipid deposition and TG homeostasis in hepatocytes is important for understanding the pathogenesis of NAFLD.

Analysis of the human miRNA database revealed no miR-122 binding sequence in the 3'UTR of FXR mRNA. Generally, the target mRNA sequence of miR-122 will become unstable and susceptible to degradation, resulting in decreased mRNA abundance and subsequent decreased translation. However, FXR was upregulated after miR-122 over-expression. Therefore, we speculated that miR-122 may indirectly induce FXR by inhibiting the expression of its repressor. Here we proposed that $Y Y 1$ could be the target of miR-122 in the liver. $Y Y 1$ is a member of the Polycomb Group protein family, which functions as a transcription factor and is important in biological processes such as development, replication and cell proliferation $[37,38]$. Recently, hepatic $Y Y 1$ expression was reported to be a critical endogenous regulator to promote TG accumulation in the liver, leading to a fatty liver. It was also proven that $Y Y 1$ promotes hepatosteatosis mainly via the repression of FXR expression [10].

In this study, we showed for the first time that miR-122 upregulates FXR expression by targeting the 3'UTR of its upstream regulator, YY1 mRNA. Moreover, the down-regulation of YY1 and consequent upregulation of FXR may play an important role in miR-122-mediated hepatic lipogenesis in vitro and in vivo. miR-122, FXR, and SHP were downregulated and YY1 was upregulated in association with lipid accumulation in vivo and in vitro. Overexpression of miR-122 suppressed the lipid content in FFA-induced steatotic hepatocytes and STZ-HFDinduced NASH mouse livers. A further study indicated that miR-122-mediated upregulation of the FXR-SHP axis by suppressing YY1 may be a key mechanism underlying its effect on the regulation of TG homeostasis.

The present study not only showed that miR-122 regulates TG homeostasis by modulating the YY1-FXR-SHP axis but also demonstrated that the effect of miR-122 downregulation on fat accumulation via the YY1-FXR-SHP axis may be involved in the pathogenesis of NAFLD. This provides a new understanding of the molecular mechanism underlying the pathogenesis of liver steatosis from a miRNA perspective.

A recent study provided strong evidence that activation of FXR upregulates miR-122 expression and, in turn, downregulates the expression of miR-122 target genes, including insulin-like growth factor-1 receptor and cyclin G1 [32]. Our study provided insight into another potential relationship between miR-122, YY1 and FXR by which miR-122 
upregulation of the FXR-SHP axis via the suppression of $Y Y 1$ may play a feedback role in hepatic TG homeostasis.

\section{Conclusion}

This is the first study to identify a novel mechanism of the regulation of $Y Y 1$ and FXR expression by miR-122 in human and mouse hepatocytes. miR-122 may play an important role in the translational control of $Y Y 1$ and FXR to regulate TG homeostasis. The overexpression of miR-122 may induce the YY1-FXR-SHP regulatory axis to reduce hepatic TG levels. miR-122 can potentially serve as a target for NAFLD treatment.

\section{Abbreviations}

miR-122 (microRNA-122); TG (Triglyceride); STZ (Streptozotocin); HFD (High-Fat Diet); NASH (Nonalcoholic Steatohepatitis); FFA (Free Fatty Acid); YY1 (Yin Yang 1); 3'UTR (3'-untranslated region); FXR (Farnesoid X Receptor); SHP (Small Heterodimer Partner); NAFLD (Nonalcoholic Fatty Liver Disease); HCC (Hepatocellular Carcinoma); SREBP-1 (Sterol Regulatory Element-binding Protein 1); LXR (Liver X Receptor); FGF21 (Fibroblast Growth Factor 21); ChREBP (Carbohydrate-responsive Element-binding Protein); APK (AMP-activated Protein Kinase).

\section{Acknowledgements}

The National Key Research and Development Program of China (grant no. 2016YFC1302600) and the Clinical Medical Center for Digestive Disease of Jiangsu Province (grant no. BL2012001).

\section{Disclosure Statement}

None.

\section{References}

1 Parekh S, Anania FA: Abnormal lipid and glucose metabolism in obesity: Implications for nonalcoholic fatty liver disease. Gastroenterology 2007;132:2191-2207.

-2 Adams LA, Lymp JF, St SJ, Sanderson SO, Lindor KD, Feldstein A, Angulo P: The natural history of nonalcoholic fatty liver disease: A population-based cohort study. Gastroenterology 2005;129:113-121.

-3 Zhang J, Zhao Y, Xu C, Hong Y, Lu H, Wu J, Chen Y: Association between serum free fatty acid levels and nonalcoholic fatty liver disease: A cross-sectional study. Sci Rep 2014;4:5832.

-4 Sanyal AJ, Campbell-Sargent C, Mirshahi F, Rizzo WB, Contos MJ, Sterling RK, Luketic VA, Shiffman ML, Clore JN: Nonalcoholic steatohepatitis: Association of insulin resistance and mitochondrial abnormalities. Gastroenterology 2001;120:1183-1192.

5 Neuschwander-Tetri BA: Hepatic lipotoxicity and the pathogenesis of nonalcoholic steatohepatitis: The central role of nontriglyceride fatty acid metabolites. Hepatology 2010;52:774-788.

6 Zeng L, Tang W, Yin J, Feng L, Li Y, Yao X, Zhou B: Alisol a 24-Acetate prevents hepatic steatosis and metabolic disorders in HepG2 cells. Cell Physiol Biochem 2016;40:453-464.

7 Jia HY, Li QZ, Lv LF. HDAC5 Inhibits Hepatic Lipogenic Genes Expression by Attenuating the Transcriptional Activity of Liver X Receptor. Cell Physiol Biochem 2016;39:1561-1567. 


\section{Cellular Physiology Cell Physiol Biochem 2017;44:1651-1664

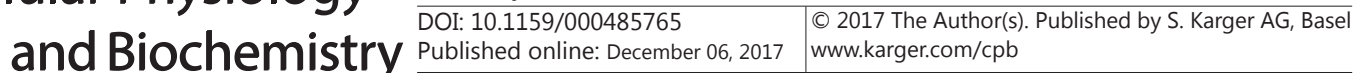

8 Asrih M, Montessuit C, Philippe J, Jornayvaz FR. Free Fatty Acids Impair FGF21 Action in HepG2 Cells. Cell Physiol Biochem 2015;37:1767-78.

-9 Wang Y, Viscarra J, Kim SJ, Sul HS: Transcriptional regulation of hepatic lipogenesis. Nat Rev Mol Cell Biol 2015;16:678-689.

10 Lu Y, Ma Z, Zhang Z, Xiong X, Wang X, Zhang H, Shi G, Xia X, Ning G, Li X: Yin Yang 1 promotes hepatic steatosis through repression of farnesoid $X$ receptor in obese mice. Gut 2014;63:170-178.

-11 Xiong X, Wang X, Lu Y, Wang E, Zhang Z, Yang J, Zhang H, Li X: Hepatic steatosis exacerbated by endoplasmic reticulum stress-mediated downregulation of FXR in aging mice. J Hepatol 2014;60:847-854.

-12 Rottiers V, Najafi-Shoushtari SH, Kristo F, Gurumurthy S, Zhong L, Li Y, Cohen DE, Gerszten RE, Bardeesy N, Mostoslavsky R, Naar AM: MicroRNAs in metabolism and metabolic diseases. Cold Spring Harb Symp Quant Biol 2011;76:225-233.

13 Davalos A, Goedeke L, Smibert P, Ramirez CM, Warrier NP, Andreo U, Cirera-Salinas D, Rayner K, Suresh U, Pastor-Pareja JC, Esplugues E, Fisher EA, Penalva LO, Moore KJ, Suarez Y, Lai EC, Fernandez-Hernando C: MiR-33a/b contribute to the regulation of fatty acid metabolism and insulin signaling. Proc Natl Acad Sci U S A 2011;108:9232-9237.

14 Liu W, Cao H, Ye C, Chang C, Lu M, Jing Y, Zhang D, Yao X, Duan Z, Xia H, Wang YC, Jiang J, Liu MF, Yan J, Ying H: Hepatic miR-378 targets p110alpha and controls glucose and lipid homeostasis by modulating hepatic insulin signalling. Nat Commun 2014;5:5684.

15 Esau C, Davis S, Murray SF, Yu XX, Pandey SK, Pear M, Watts L, Booten SL, Graham M, McKay R, Subramaniam A, Propp S, Lollo BA, Freier S, Bennett CF, Bhanot S, Monia BP: MiR-122 regulation of lipid metabolism revealed by in vivo antisense targeting. Cell Metab 2006;3:87-98.

-16 Elmen J, Lindow M, Schutz S, Lawrence M, Petri A, Obad S, Lindholm M, Hedtjarn M, Hansen HF, Berger U, Gullans S, Kearney P, Sarnow P, Straarup EM, Kauppinen S: LNA-mediated microRNA silencing in nonhuman primates. Nature 2008;452:896-899.

-17 Bandiera S, Pfeffer S, Baumert TF, Zeisel MB: MiR-122--a key factor and therapeutic target in liver disease. J Hepatol 2015;62:448-457.

18 Nie YQ, Cao J, Zhou YJ, Liang X, Du YL, Wan YJ, Li YY: The effect of miRNA-122 in regulating fat deposition in a cell line model. J Cell Biochem 2014;115:839-846.

19 Gatfield D, Le Martelot G, Vejnar CE, Gerlach D, Schaad O, Fleury-Olela F, Ruskeepää AL, Oresic M, Esau CC, Zdobnov EM, Schibler U.l: Integration of microRNA miR-122 in hepatic circadian gene expression. Genes Dev 2009;23:1313-1326.

20 Akazawa Y, Cazanave S, Mott JL, Elmi N, Bronk SF, Kohno S, Charlton MR, Gores GJ: Palmitoleate attenuates palmitate-induced Bim and PUMA up-regulation and hepatocyte lipoapoptosis. J Hepatol 2010;52:586-593.

21 Gomez-Lechon MJ, Donato MT, Martinez-Romero A, Jimenez N, Castell JV, O'Connor JE: A human hepatocellular in vitro model to investigate steatosis. Chem Biol Interact 2007;165:106-116.

-22 Fujii M, Shibazaki Y, Wakamatsu K, Honda Y, Kawauchi Y, Suzuki K, Arumugam S, Watanabe K, Ichida T, Asakura H, Yoneyama H: A murine model for non-alcoholic steatohepatitis showing evidence of association between diabetes and hepatocellular carcinoma. Med Mol Morphol 2013;46:141-152.

-23 Wobser H, Dorn C, Weiss TS, Amann T, Bollheimer C, Buttner R, Scholmerich J, Hellerbrand C: Lipid accumulation in hepatocytes induces fibrogenic activation of hepatic stellate cells. Cell Res 2009;19:9961005.

24 Wang H, Xie Z, Hou T, Li Z, Huang K, Gong J, Zhou W, Tang K, Xu J, Dong S: MiR-125b regulates the osteogenic differentiation of human mesenchymal stem cells by targeting BMPR1b. Cell Physiol Biochem 2017;41:530542.

25 Huang MQ Zhou CJ, Zhang YP, Zhang XQ Xu W, Lin J, Wang PJ: Salvianolic acid b ameliorates hyperglycemia and dyslipidemia in db/db mice through the AMPK pathway. Cell Physiol Biochem 2016;40:933-943.

26 Kleiner DE, Brunt EM, Van Natta M, Behling C, Contos MJ, Cummings OW, Ferrell LD, Liu YC, Torbenson MS, Unalp-Arida A, Yeh M, McCullough AJ, Sanyal AJ: Design and validation of a histological scoring system for nonalcoholic fatty liver disease. Hepatology 2005;41:1313-1321.

27 Ratziu V, Goodman Z, Sanyal A: Current efforts and trends in the treatment of NASH. J Hepatol 2015;62:S65-S75.

-28 Szabo G, Bala S: MicroRNAs in liver disease. Nat Rev Gastroenterol Hepatol 2013;10:542-552. 


\section{Cellular Physiology Cell Physiol Biochem 2017;44:1651-1664 \begin{tabular}{l|l|l} 
DOI: 10.1159/000485765 & $\begin{array}{l}\text { O 2017 The Author(s). Published by S. Karger AG, Basel } \\
\text { www.karger.com/cpb }\end{array}$
\end{tabular}}

Wu et al.: Regulation of Hepatic Triglyceride by MicroRNA-122

-29 Xu H, He JH, Xiao ZD, Zhang QQ, Chen YQ, Zhou H, Qu LH: Liver-enriched transcription factors regulate microRNA-122 that targets CUTL1 during liver development. Hepatology 2010;52:1431-1442.

-30 Laudadio I, Manfroid I, Achouri Y, Schmidt D, Wilson MD, Cordi S, Thorrez L, Knoops L, Jacquemin P, Schuit F, Pierreux CE, Odom DT, Peers B, Lemaigre FP: A feedback loop between the liver-enriched transcription factor network and miR-122 controls hepatocyte differentiation. Gastroenterology 2012;142:119-129.

-31 Deng XG, Qiu RL, Wu YH, Li ZX, Xie P, Zhang J, Zhou JJ, Zeng LX, Tang J, Maharjan A, Deng JM: Overexpression of miR-122 promotes the hepatic differentiation and maturation of mouse ESCs through a miR-122/FoxA1/ HNF4a-positive feedback loop. Liver Int 2014;34:281-295.

-32 He J, Zhao K, Zheng L, Xu Z, Gong W, Chen S, Shen X, Huang G, Gao M, Zeng Y, Zhang Y, He F: Upregulation of microRNA-122 by farnesoid $X$ receptor suppresses the growth of hepatocellular carcinoma cells. Mol Cancer 2015;14:163.

33 Sinal CJ, Tohkin M, Miyata M, Ward JM, Lambert G, Gonzalez FJ: Targeted disruption of the nuclear receptor FXR/BAR impairs bile acid and lipid homeostasis. Cell 2000;102:731-744.

34 Zhang Y, Lee FY, Barrera G, Lee H, Vales C, Gonzalez FJ, Willson TM, Edwards PA: Activation of the nuclear receptor FXR improves hyperglycemia and hyperlipidemia in diabetic mice. Proc Natl Acad Sci U S A 2006;103:1006-1011.

35 Rotman Y, Sanyal AJ: Current and upcoming pharmacotherapy for non-alcoholic fatty liver disease. Gut 2017;66:180-190.

-36 Mudaliar S, Henry RR, Sanyal AJ, Morrow L, Marschall HU, Kipnes M, Adorini L, Sciacca CI, Clopton P, Castelloe E, Dillon P, Pruzanski M, Shapiro D: Efficacy and safety of the farnesoid X receptor agonist obeticholic acid in patients with type 2 diabetes and nonalcoholic fatty liver disease. Gastroenterology 2013;145:574-582.

-37 Deng Z, Cao P, Wan MM, Sui G: Yin Yang 1: A multifaceted protein beyond a transcription factor. Transcription 2010;1:81-84.

-38 Castellano G, Torrisi E, Ligresti G, Malaponte G, Militello L, Russo AE, McCubrey JA, Canevari S, Libra M: The involvement of the transcription factor Yin Yang 1 in cancer development and progression. Cell Cycle 2009;8:1367-1372. 